\title{
OPEN Changes of antibiotic prescribing pattern and its resistance to $E$. Coli in South Korea: a 12-year retrospective observational study
}

\author{
Geun Woo Lee ${ }^{1,4}$, Sukhyun Ryu ${ }^{2,4}$, Juhee Park ${ }^{1}$, Eun Jee Lee ${ }^{1}$, Kwang Jun Lee ${ }^{3}$, \\ Jungyeon $\mathrm{Tae}^{2}$, Youngsik Hwang ${ }^{2}$ \& Dong-Sook Kim ${ }^{1 \bowtie}$
}

In the present study, we investigated the pattern of changes in antibiotic prescription and antimicrobial resistance (AMR) in Escherichia coli in South Korea between 2007 and 2018. We collected data related to antibiotic prescription and AMR in $E$. coli from the national surveillance system. We used the Mann-Kendall test and Spearman's correlation to identify the trends of antibiotic prescription and AMR in E. coli and to examine the relationship between them, respectively. Although we noted a significant decreasing trend of ampicillin and gentamicin prescriptions in all medical institutions, we identified a higher level of AMR in long-term care facilities than in other medical institutions. We did not identify a significant positive correlation between ampicillin and gentamicin prescriptions and their resistance in $E$. coli. However, we found a significant positive correlation between cefotaxime prescription and its resistance in $E$. coli in hospitals, long-term care facilities, and clinics. Our results strongly suggest that long-term care facilities in South Korea have the potential to sustain AMR epidemics and that more efforts are needed to curb AMR in $E$. coli. Further epidemiological studies using enhanced AMR surveillance are warranted.

The development of antimicrobial resistance (AMR) is a normal evolutionary process in microorganisms. It is often accelerated by selective pressure exerted by the overuse or misuse of antimicrobial agents ${ }^{1}$. The increasing use of antibiotics has led to the emergence of AMR, which limits the treatment of common bacterial infections. Gram-negative bacteria, such as Escherichia coli, which are resistant to third-generation cephalosporins, are particularly of great concern. This resistance is conferred by the production of extended-spectrum beta-lactamases (ESBLs) and is transferred to other gram-negative bacteria; therefore, the surveillance of AMR in E. coli is often used as a proxy for the surveillance of ESBLs in humans, animals, and the environment ${ }^{2}$.

South Korea is an East Asian country with a population of 51.4 million. In South Korea, broad-spectrum antibiotics, including quinolone, penicillin, and cephalosporin are frequently prescribed ${ }^{3,4}$. In the twenty-first century, extensive efforts have been made in South Korea to reduce AMR. These include the Korean National Antimicrobial Resistance Safety Control Program led by the Korean Ministry of Food and Drug Safety from 2003 to $2013^{5}$ and the Korean national action plan on AMR led by the Korean Ministry of Health and Welfare from 2016 to $2020^{6}$. To identify the level of antibiotic consumption and AMR within a country, population-based studies have mainly been conducted in countries in the UK and USA ${ }^{7,8}$. Different healthcare settings and public health policies in each country would affect the changes in antibiotic prescription because these factors are very likely to affect the selection pressures exerted on microorganisms in the population. Therefore, surveillance and research on changes in antibiotic consumption and AMR with changes in related health policies in different countries are important in order to develop evidence-based strategies to overcome AMR $^{6}$. However, there exists a knowledge gap regarding the level of antibiotic prescription and AMR in Asia, which is highly vulnerable to AMR threats ${ }^{9}$.

To the best of our knowledge, no long-term observational study has been conducted in South Korea to assess the frequency of antibiotic prescription and AMR considering the changes in healthcare regulation. In the present

\footnotetext{
${ }^{1}$ Pharmaceutical and Medical Technology Research Team, Department of Research, Health Insurance Review and Assessment Service, Wonju, South Korea. ${ }^{2}$ Department of Preventive Medicine, Konyang University College of Medicine, Daejeon, South Korea. ${ }^{3}$ National Institute of Health, Korean Centers for Disease Control and Prevention, Osong, South Korea. ${ }^{4}$ These authors contributed equally: Geun Woo Lee and Sukhyun Ryu. 更email: sttone@hira.or.kr
} 


\begin{tabular}{|l|l|l|l|l|l|}
\hline DID & General hospitals & Hospitals & LTCFs & Clinics & Overall \\
\hline Fluoroquinolone & $4.00(3.60-4.32)$ & $0.91(0.78-0.99)$ & $1.19(0.75-2.47)$ & $1.79(1.33-2.15)$ & $7.90(6.46-9.93)$ \\
\hline Ampicillin & $0.97(0.64-1.12)$ & $0.94(0.65-1.16)$ & $0.57(0.33-1.18)$ & $2.12(1.24-2.91)$ & $4.60(2.85-6.36)$ \\
\hline Cefotaxime & $5.04(5.34-4.58)$ & $0.83(0.49-1.15)$ & $1.28(0.86-1.73)$ & $0.59(0.29-0.97)$ & $7.75(6.21-9.19)$ \\
\hline Gentamicin & $0.03(0.02-0.06)$ & $0.04(0.03-0.07)$ & $0.08(0.06-0.22)$ & $0.08(0.06-0.10)$ & $0.23(0.15-0.44)$ \\
\hline
\end{tabular}

Table 1. Antibiotic prescription in South Korea between 2007 and 2018. Each value in the cell indicates the mean DID and the range (min-max). DID defined daily dose/1000 inhabitants/day, LTCFs long-term care facilities.
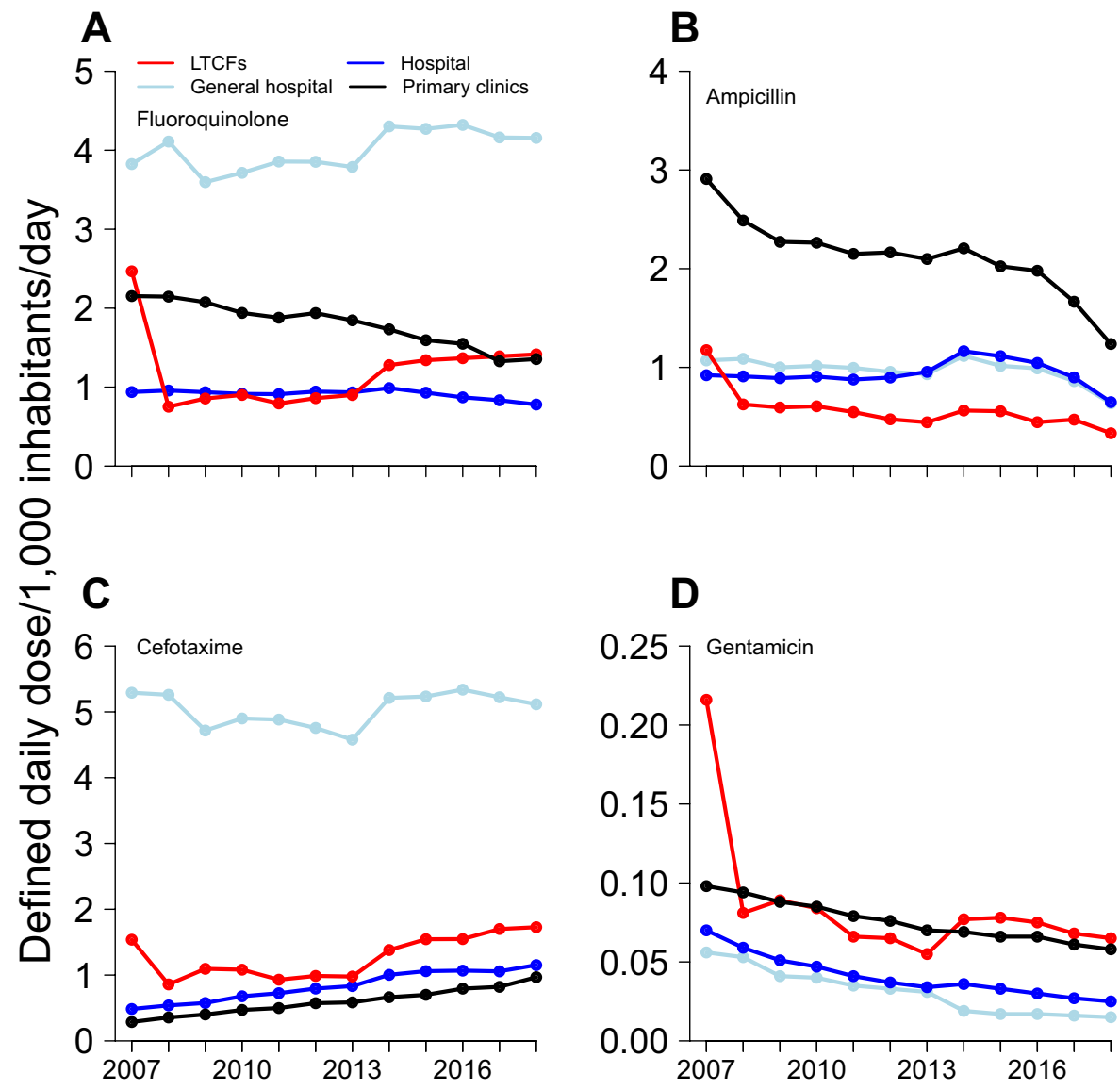

\section{D}

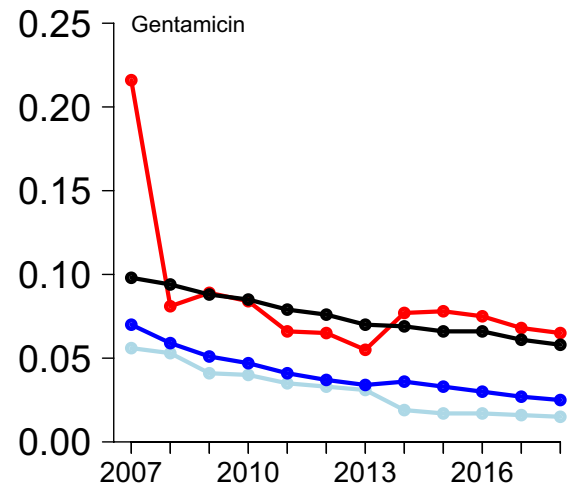

Years

Figure 1. Prescription trend of antibiotics, including (A) fluoroquinolone, (B) ampicillin, (C) cefotaxime and (D) gentamicin, in South Korea between 2007 and 2018. The red line indicates long-term care facilities (LTCFs), and the blue line indicates hospitals. The white-blue line indicates general hospitals, and the black line indicates primary clinics.

study, we used the South Korean national surveillance data of 12 years to focus on broad-spectrum antimicrobial agents and their resistance in E. coli.

\section{Results}

The overall mean DID of fluoroquinolone and cefotaxime during the study period was 7.90 (range, 6.46-9.93) and 7.75 (6.21-9.19), respectively (Table 1). These antibiotics were prescribed more commonly in general hospitals than in other medical institutions. The overall mean DID of ampicillin and gentamicin was $4.60(2.85-6.36)$ and 0.23 (0.15-0.44), respectively. Ampicillin was mostly prescribed in clinics (DID, 0.59; range, 1.24-2.91), while gentamicin was mostly prescribed in LTCFs (DID, 0.08; range, 0.06-0.22). We observed a decreasing trend of fluoroquinolone prescription in hospitals $(\tau=-0.52, p=0.02)$ and clinics $(\tau=-0.94, p<0.01)$ (Fig. 1 , Supplementary Table 1). Furthermore, we observed a decreasing trend of ampicillin and gentamicin prescriptions in 


\begin{tabular}{|l|l|l|l|l|}
\hline Antibiotic to which E. coli was resistant & General hospitals & Hospitals & LTCFs & Clinics \\
\hline Fluoroquinolone & $39.54(32.00-47.80)$ & $50.33(44.80-58.40)$ & $75.97(66.80-85.60)$ & $32.44(23.20-41.40)$ \\
\hline Ampicillin & $68.51(65.20-71.20)$ & $73.26(71.20-74.60)$ & $84.94(82.40-88.80)$ & $63.81(62.50-66.10)$ \\
\hline Cefotaxime & $27.98(13.00-38.60)$ & $31.94(22.70-41.00)$ & $54.24(36.770-70.50)$ & $15.13(4.70-23.10)$ \\
\hline Gentamicin & $28.19(26.60-29.60)$ & $32.82(30.60-36.90)$ & $43.36(40.20-47.40)$ & $25.08(22.90-27.10)$ \\
\hline
\end{tabular}

Table 2. Antibiotic resistance rate of Escherichia coli in South Korea between 2007 and 2018. Each value in the cell indicates the mean antimicrobial resistance rate and the range (min-max). LTCFs long-term care facilities.
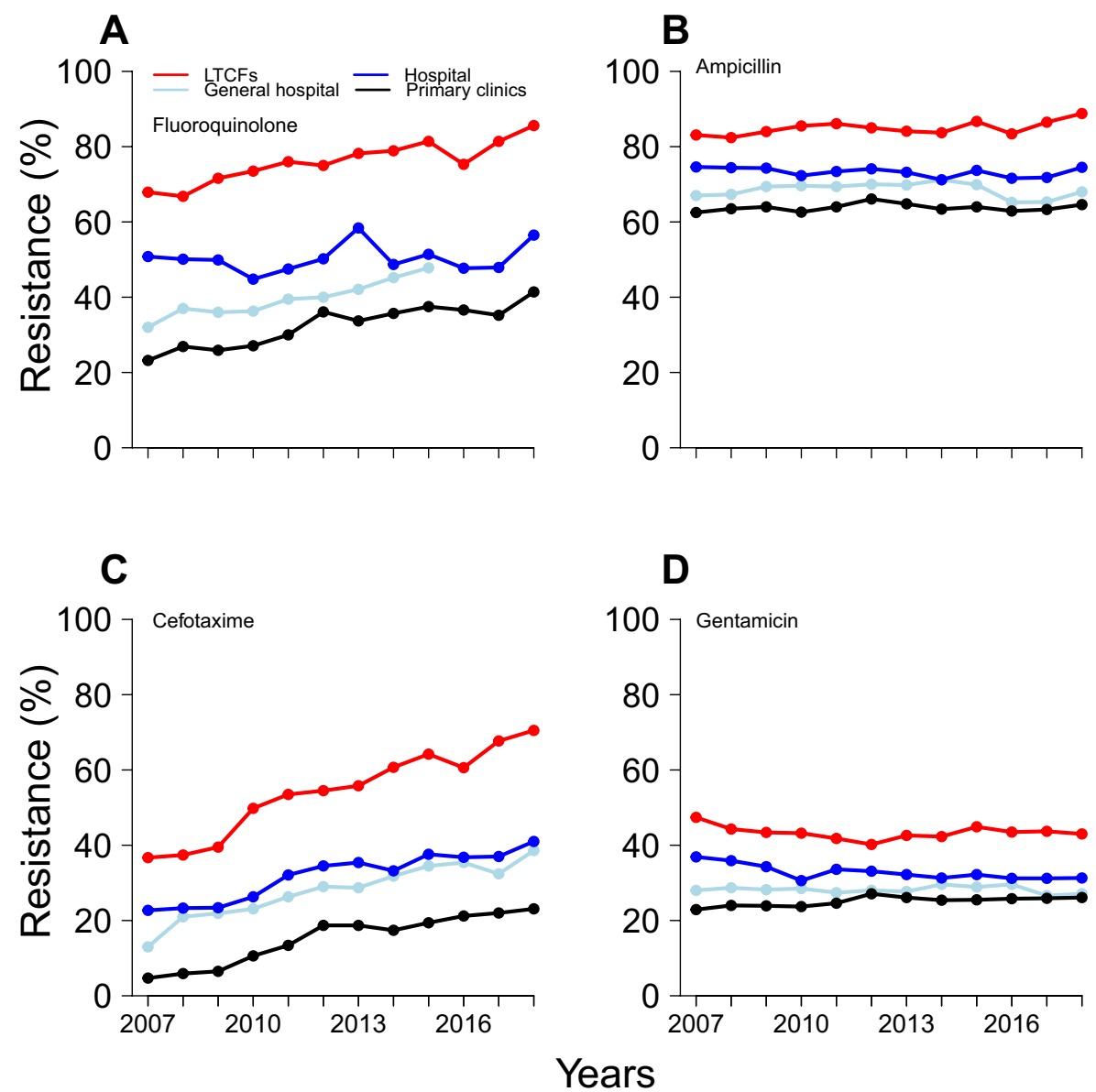

Figure 2. Resistance rate of Escherichia coli to antibiotics, including (A) fluoroquinolone, (B) ampicillin, (C) cefotaxime and (D) gentamicin, in South Korea between 2007 and 2018. The red line indicates long-term care facilities (LTCFs), and the blue line indicates hospitals. The white-blue line indicates general hospitals, and the black line indicates primary clinics.

all medical institutions (Fig. 1, Supplementary Table 1). However, we noted an increasing trend of cefotaxime prescription in hospitals $(\tau=0.94, p<0.01)$, LTCFs $(\tau=0.55, p=0.02)$ and clinics $(\tau=1.0, p<0.01)$.

The mean resistance rate of $E$. coli was the highest in LTCFs (fluoroquinolone $76 \%$, ampicillin $85 \%$, cefotaxime $54 \%$ and gentamicin 43\%) (Table 2) and the lowest in clinics (fluoroquinolone 32\%, ampicillin 64\%, cefotaxime $15 \%$ and gentamicin 25\%) (Table 2). We observed an increasing trend of fluoroquinolone resistance in $E$. coli in general hospitals $(\tau=0.89, p<0.01)$, LTCFs $(\tau=0.81, p<0.01)$ and clinics $(\tau=0.76, p<0.01)$. Moreover, we observed a significant increasing trend of cefotaxime resistance in E. coli in all medical institutions (Fig. 2, Supplementary Table 2). Furthermore, we identified increasing and decreasing trends of gentamicin resistance in $E$. coli in clinics $(\tau=0.60, p<0.01)$ and hospitals $(\tau=-0.64, p<0.01)$, respectively.

We observed a negative correlation between fluoroquinolone and gentamicin prescriptions and their resistance in E. coli in clinics (fluoroquinolone-0.82, gentamicin-0.73); however, we noted a positive correlation between gentamicin prescription and its resistance in $E$. coli in hospitals $(\tau=0.69, p=0.01)$ and LTCFs $(\tau=0.65$, $p=0.02$ ) (Table 3). Moreover, we identified a positive correlation between cefotaxime prescription and its resistance in $E$. coli in hospitals $(\tau=0.96, p<0.01)$, LTCFs $(\tau=0.66, p=0.02)$ and clinics $(\tau=0.98, p<0.01)$. 


\begin{tabular}{|l|l|l|l|l|}
\hline Antibiotic to which $\boldsymbol{E}$. coli was resistant & General hospitals & Hospitals & LTCFs & Clinics \\
\hline Fluoroquinolone & $0.67(0.06)$ & $0.17(0.60)$ & $0.4(0.15)$ & $-0.82(<0.01)$ \\
\hline Ampicillin & $0.24(0.46)$ & $-0.46(0.13)$ & $-0.53(0.08)$ & $-0.31(0.32)$ \\
\hline Cefotaxime & $0.13(0.68)$ & $0.96(<0.01)$ & $0.66(0.02)$ & $0.98(<0.01)$ \\
\hline Gentamicin & $0.16(0.61)$ & $0.69(0.01)$ & $0.65(0.02)$ & $-0.73(<0.01)$ \\
\hline
\end{tabular}

Table 3. Correlation analysis of antibiotic prescription and resistance in Escherichia coli by the institutions between 2007 and 2018. Each value in the cell indicates the coefficient and p-value. LTCFs long-term care facilities.

\section{Discussion}

The increasing use of antibiotics is a key factor contributing to the increased risk of $\mathrm{AMR}^{10}$. In the present study, we identified an increasing trend of cefotaxime prescription and of fluoroquinolone and cefotaxime resistance in E. coli. Furthermore, we identified a decreasing trend of fluoroquinolone prescriptions in hospitals and clinics and a decreasing trend of ampicillin and gentamicin prescriptions in all medical institutions.

A higher level of fluoroquinolone and cefotaxime was prescribed in general hospitals than in hospitals, which may be due to the severity of diseases in patients admitted there. Although fluoroquinolone and cefotaxime were more frequently prescribed in general hospitals, their resistance in E. coli was higher in LTCFs. This is likely because elderly patients usually visit general hospitals or hospitals for receiving medication rather than visiting LTCFs. Furthermore, AMR epidemics in LTCFs possibly affected the study results ${ }^{11}$.

The National Antimicrobial Resistance Safety Control Program implemented from 2003 to 2013 mainly included basic microbial research, AMR surveillance, and infection control ( $96 \%$ of the overall investment $)^{5}$. However, this program could not extensively reduce AMR in E. coli during our study period.

The 5-year Korean action plan on AMR implemented in 2016 included the quality assessment of antibiotics used in medical institutions and improvement of the surveillance system of AMR ${ }^{6}$. The HIRA implemented a programme to assess the benefits of antibiotics prescribed for common cold during the study period and reported a $14 \%$ decrease in the number of antibiotics prescribed for common cold in clinics (from 54\% in 2006 to $40 \%$ in 2017). This programme may largely reduce the prescription of beta-lactam antibiotics, which are the most commonly prescribed antibiotics in primary clinics in South Korea ${ }^{4}$. Furthermore, the large-scale public awareness campaign for the appropriate consumption of antibiotics may reduce the prescription of beta-lactam antibiotics in clinics ${ }^{12}$.

We identified a positive correlation between the prescription of fluoroquinolone and gentamicin and their resistance in E.coli in hospitals and LTCFs which is consistent with findings from other studies ${ }^{13-15}$. However, we identified a negative correlation in clinics. This result may be due to the decreasing trend of fluoroquinolone and gentamicin prescription in the clinic which was likely to be affected by the quality assessment program for antibiotic prescription in clinics from HIRA.

We also identified a negative correlation between ampicillin prescription and its resistance in E. coli. This result may be affected by the transmission of ESBLs, which confer resistance to third-generation cephalosporins, from $E$. coli and the resultant resistance to other beta-lactam antibiotics, including ampicillin ${ }^{2,10}$.

We found the highest AMR in LTCFs during our study period. Although antibiotic prescription is not very high in LTCFs in South Korea, most prescribed antibiotics are broad-spectrum, similar to the findings of a previous study ${ }^{16}$. Furthermore, AMR is higher in LTCFs than in the general community. This is in accordance with previous findings of higher AMR in elderly residents in LTCFs than in the general population ${ }^{17,18}$. This is likely due to the fact that most residents in LTCFs are elderly people with multiple chronic diseases and medications ${ }^{19}$ and with an increased risk of acquiring infections and developing AMR $^{20-22}$. Very few studies have examined antibiotic prescription and resistance in LTCFs because of the characteristics of the resident population and the difficulty in forming a control group in the general population. Therefore, a study focusing on enhanced surveillance in LTCFs is needed to identify the risk factors for AMR and to measure the burden of AMR and the impact of AMR epidemic measures ${ }^{11}$.

There are several limitations of the present study. First, we did not include detailed data on demographic factors and comorbidities; therefore, we could not identify the risk factors for antibiotic prescription and AMR. Second, we did not include the appropriateness of antibiotic prescription, which would indicate the overuse of antibiotics. Third, we used DID as the indicator of antibiotic prescription rather than days of therapy or beds; however, this indicator has been widely used to monitor the trend of antibiotic prescription at the population level.

Fourth, we collected data regarding resistance in E. coli from the pathogen-based surveillance, which may limit the interpretation of $\mathrm{AMR}^{23}$. However, the present study provides insights into antibiotic prescription in South Korea, which may help in developing national antimicrobial stewardship. Enhanced surveillance of AMR, e.g. case-based surveillance, may overcome these limitations and help reveal the impact of AMR-related public health policies ${ }^{23}$.

In the present study, we identified a decreasing trend of ampicillin prescription but an increasing trend of cefotaxime prescription and cefotaxime resistance in E. coli between 2007 and 2018. Further research based on enhanced surveillance of AMR to measure the impact of AMR-related policies in South Korea is warranted. 


\section{Methods}

In this retrospective study, anonymised data regarding antibiotic prescription and AMR were extracted from the publicly available national database and laboratory-based surveillance system, respectively ${ }^{24,25}$. The study was approved and informed consent was waived by the Institutional Review Board of the Korean Health Insurance Review and Assessment Service (HIRA) (IRB No. 2017004-002). All methods were carried out in accordance with relevant guidelines from HIRA.

Antibiotic prescription. We collected the data of antibiotic prescription from the nation-wide population-based database. The Korean National Health Insurance provides near-complete coverage of all antibiotic prescriptions in South Korea (approximately $98 \%$ of the total Korean population) ${ }^{4}$. All Korean medical institutions including general hospitals, hospitals, LTCFs, and clinics submit claims to the HIRA. The submitted medical claims include the name of the diagnosis, drug code, drug brand name, active ingredient, route of administration, amount in a single dose, daily dose, total number of days of administration or number of doses administered and prescription date. The administrative medical claims data collected from the HIRA were used for analysing the antibiotic prescriptions. The therapeutic drug class was determined using the World Health Organization's Anatomical Therapeutic Chemical (ATC) classification system.

The antibiotics were classified into ATC code J01 (antibacterials for systemic use). Of the drugs registered between 2007 and 2018, a total of 3445 antibacterial agents were listed under ATC code J01. Analysis by class was performed for ATC levels 3 and 4, where level 3 corresponds to pharmacological and therapeutic properties and level 4 corresponds to chemical structures.

To measure the standardised level of antibiotic prescription across various antibiotic products, annual antibiotic prescriptions were calculated as the defined daily dose (DDD)/1000 inhabitants/day. This indicator was widely used to examine the relation between antibiotic prescription and AMR on population-level ${ }^{26,27}$. The annual population between 2007 and 2018 was acquired from Statistics Korea ${ }^{28}$.

$$
\mathrm{DDD} / 1000 \text { inhabitants } / \text { day }=\frac{\sum X i j(\mathrm{mg}) \times 1000 \text { persons }}{\mathrm{DDDi}(\mathrm{mg}) \times 365 \times \mathrm{Pi}}
$$

X: drug use, P: number of people, i: population category (gender, age and facilities), j: ATC.

Antimicrobial resistance. We collected the resistance rate from the Korean Antimicrobial Resistance Monitoring System (KARMS) operated by the Korea Centers for Disease Control and Prevention (KCDC) ${ }^{29}$. This national AMR monitoring system is laboratory-based sentinel surveillance which collected laboratory data from the KCDC designated hospitals and laboratories where performed KCDC approved antimicrobial susceptibility tests ${ }^{30}$. In South Korea, the physicians in the primary clinics and LTCFs usually examined the clinical samples of their patients at the KCDC designated laboratories. The sentinel institutions including hospitals and laboratories report to KARMS the AMR rate which was calculated using the arithmetic mean values of the clinical sample obtained from hospitals, LTCFs, and clinics. This sentinel surveillance of AMR has been widely used for tracking the proportions of resistant isolates in the country level ${ }^{31}$. From 2016, the KCDC-designated laboratory institutions examined the specimens collected from the sentinel institutions.

Definitions. General hospitals and hospitals were defined as medical institutions with more than 100 beds and 30-100 beds, respectively. LTCFs were defined as institutions that mainly provides rehabilitative care for the elderly. Clinics were defined as institutions with less than 30 beds that mainly provided outpatient care ${ }^{25}$.

Statistical analysis. We used the Mann-Kendall test to identify the trends of antibiotic prescription and AMR in E. coli and used Spearman's correlation to examine the relationship between them. In the present study, we excluded the prescription data of 2007 from long-term care facilities (LTCFs), as some institutions had outlier data. Moreover, fluoroquinolone resistance rates of $E$. coli were not measured in general hospitals from 2016 to 2018. Analyses were performed using R version 3.6.1 (R Foundation for Statistical Computing, Vienna, Austria).

Ethics approval. The present study was approved by the Institutional Review Board of the Korean Health Insurance Review and Assessment Service (HIRA) (IRB No. 2017004-002). The informed consent was waived by the Institutional Review Board of HIRA.

\section{Data availability}

All data used within the study are available from the corresponding author on request.

Received: 28 October 2020; Accepted: 4 February 2021

Published online: 11 March 2021

\section{References}

1. Hughes, D. Selection and evolution of resistance to antimicrobial drugs. IUBMB Life 66, 521-529. https://doi.org/10.1002/iub.1278 (2014).

2. Mir, R. A. et al. Identification and characterization of cefotaxime resistant bacteria in beef cattle. PLoS ONE 11, e0163279. https ://doi.org/10.1371/journal.pone.0163279 (2016).

3. Park, J., Han, E., Lee, S. O. \& Kim, D. S. Antibiotic use in South Korea from 2007 to 2014: a health insurance database-generated time series analysis. PLoS ONE 12, e0177435. https://doi.org/10.1371/journal.pone.0177435 (2017). 
4. Ryu, S. et al. Temporal relationship between antibiotic use and respiratory virus activities in the Republic of Korea: a time-series analysis. Antimicrob. Resist. Infect. Control 7, 56. https://doi.org/10.1186/s13756-018-0347-8 (2018).

5. Ryu, S., Head, M. G., Kim, B. I., Hwang, J. \& Cho, E. H. Are we investing wisely? A systematic analysis of nationally funded antimicrobial resistance projects in Republic of Korea, 2003-2013. J. Glob. Antimicrob. Resist. 6, 90-94. https://doi.org/10.1016/j. jgar.2016.03.007 (2016).

6. Ryu, S. The new Korean action plan for containment of antimicrobial resistance. J. Glob. Antimicrob. Resist. 8, 70-73. https://doi. org/10.1016/j.jgar.2016.10.013 (2017).

7. Cars, O., Molstad, S. \& Melander, A. Variation in antibiotic use in the European Union. Lancet 357, 1851-1853. https://doi. org/10.1016/S0140-6736(00)04972-2 (2001).

8. Patrick, D. M. et al. Per capita antibiotic consumption: how does a North American jurisdiction compare with Europe?. Clin. Infect. Dis. 39, 11-17. https://doi.org/10.1086/420825 (2004).

9. Yam, E. L. Y. et al. Antimicrobial resistance in the Asia Pacific region: a meeting report. Antimicrob. Resist. Infect. Control 8, 202. https://doi.org/10.1186/s13756-019-0654-8 (2019).

10. Ryu, S., Klein, E. Y. \& Chun, B. C. Temporal association between antibiotic use and resistance in Klebsiella pneumoniae at a tertiary care hospital. Antimicrob. Resist. Infect. Control 7, 83. https://doi.org/10.1186/s13756-018-0373-6 (2018).

11. van den Dool, C., Haenen, A., Leenstra, T. \& Wallinga, J. The role of nursing homes in the spread of antimicrobial resistance over the healthcare network. Infect. Control Hosp. Epidemiol. 37, 761-767. https://doi.org/10.1017/ice.2016.59 (2016).

12. Chaintarli, K. et al. Impact of a United Kingdom-wide campaign to tackle antimicrobial resistance on self-reported knowledge and behaviour change. BMC Public Health 16, 393. https://doi.org/10.1186/s12889-016-3057-2 (2016).

13. Stapleton, A. E., Wagenlehner, F. M. E., Mulgirigama, A. \& Twynholm, M. Escherichia coli resistance to fluoroquinolones in community-acquired uncomplicated urinary tract infection in women: a systematic review. Antimicrob. Agents Chemother. https ://doi.org/10.1128/AAC.00862-20 (2020).

14. Jensen, V. F., Jakobsen, L., Emborg, H. D., Seyfarth, A. M. \& Hammerum, A. M. Correlation between apramycin and gentamicin use in pigs and an increasing reservoir of gentamicin-resistant Escherichia coli. J. Antimicrob. Chemother. 58, 101-107. https://doi. org/10.1093/jac/dkl201 (2006).

15. Livermore, D. M. et al. Trends in fluoroquinolone (ciprofloxacin) resistance in enterobacteriaceae from bacteremias, England and Wales, 1990-1999. Emerg. Infect. Dis. 8, 473-478. https://doi.org/10.3201/eid0805.010204 (2002).

16. Stepan, D. et al. Antimicrobial prescribing in long-term care facilities: a nationwide point-prevalence study, Slovenia, 2016. Eur. Surveill. https://doi.org/10.2807/1560-7917.ES.2018.23.46.1800100 (2018).

17. Rosello, A. et al. Impact of long-term care facility residence on the antibiotic resistance of urinary tract Escherichia coli and Klebsiella. J. Antimicrob. Chemother. 72, 1184-1192. https://doi.org/10.1093/jac/dkw555 (2017).

18. Marra, F. et al. Utilization of antibiotics in long-term care facilities in British Columbia, Canada. J. Am. Med. Dir. Assoc. 18(1098), e1091-e1098 e1011. https://doi.org/10.1016/j.jamda.2017.09.018 (2017).

19. Kim, H. A., Shin, J. Y., Kim, M. H. \& Park, B. J. Prevalence and predictors of polypharmacy among Korean elderly. PLoS ONE 9, e98043. https://doi.org/10.1371/journal.pone.0098043 (2014).

20. Gavazzi, G. \& Krause, K. H. Ageing and infection. Lancet Infect. Dis. 2, 659-666. https://doi.org/10.1016/s1473-3099(02)00437-1 (2002).

21. Adam, H. J. et al. Comparison of pathogens and their antimicrobial resistance patterns in paediatric, adult and elderly patients in Canadian hospitals. J. Antimicrob. Chemother. 68(Suppl 1), i31-i37. https://doi.org/10.1093/jac/dkt024 (2013).

22. Sanchez, G. V. et al. Antibiotic resistance among urinary isolates from female outpatients in the United States in 2003 and 2012. Antimicrob. Agents Chemother. 60, 2680-2683. https://doi.org/10.1128/AAC.02897-15 (2016).

23. Ryu, S. et al. Case-based surveillance of antimicrobial resistance with full susceptibility profiles. JAC Antimicrob. Resist. 1, dlz070. https://doi.org/10.1093/jacamr/dlz070 (2019).

24. Korea Centers for Disease Control and Prevention. One Health AMR http://www.cdc.go.kr/nohas (2020).

25. Korea Centers for Disease Control and Prevention. Korea Antimicrobial Resistance Monitoring System 2016 (Korea Centers for Disease Control and Prevention, 2018).

26. Wojkowska-Mach, J. et al. Antibiotic consumption and antimicrobial resistance in Poland; findings and implications. Antimicrob. Resist. Infect. Control 7, 136. https://doi.org/10.1186/s13756-018-0428-8 (2018).

27. Kolozsvari, L. R. et al. Patient-related factors, antibiotic prescribing and antimicrobial resistance of the commensal Staphylococcus aureus and Streptococcus pneumoniae in a healthy population-Hungarian results of the APRES study. BMC Infect. Dis. 19, 253. https://doi.org/10.1186/s12879-019-3889-3 (2019).

28. Korea Statistics. Population in South Korea http://kosis.kr/conts/nsportalStats/nsportalStats_0102Body.jsp?menuId=10 (2020).

29. Kim, D. et al. Increasing resistance to extended-spectrum cephalosporins, fluoroquinolone, and carbapenem in gram-negative bacilli and the emergence of carbapenem non-susceptibility in Klebsiella pneumoniae: analysis of Korean antimicrobial resistance monitoring system (KARMS) data from 2013 to 2015. Ann. Lab. Med. 37, 231-239. https://doi.org/10.3343/alm.2017.37.3.231 (2017).

30. Liu, C. et al. Antimicrobial resistance in South Korea: a report from the Korean global antimicrobial resistance surveillance system (Kor-GLASS) for 2017. J. Infect. Chemother. 25, 845-859. https://doi.org/10.1016/j.jiac.2019.06.010 (2019).

31. Schrag, S. J., Zell, E. R., Schuchat, A. \& Whitney, C. G. Sentinel surveillance: a reliable way to track antibiotic resistance in communities?. Emerg. Infect. Dis. 8, 496-502. https://doi.org/10.3201/eid0805.010268 (2002).

\section{Acknowledgements}

S.R. was supported by the National Research Foundation of Korea funded by the Ministry of Education (NRF-2018R1A6A3A03012236).

\section{Author contributions}

D.K. and S.R. conceived, designed, and guided the study; G.W.L., S.R., J.P., E.J.L., K.J.L, J.T., Y.H., and D.K. analyzed the data. G.W.L., S.R. and D.K. wrote the first draft. All authors contributed to the final draft.

\section{Competing interests}

The authors declare no competing interests.

\section{Additional information}

Supplementary Information The online version contains supplementary material available at https://doi. org/10.1038/s41598-021-84450-z.

Correspondence and requests for materials should be addressed to D.-S.K. 
Reprints and permissions information is available at www.nature.com/reprints.

Publisher's note Springer Nature remains neutral with regard to jurisdictional claims in published maps and institutional affiliations.

(c) (i) Open Access This article is licensed under a Creative Commons Attribution 4.0 International License, which permits use, sharing, adaptation, distribution and reproduction in any medium or format, as long as you give appropriate credit to the original author(s) and the source, provide a link to the Creative Commons licence, and indicate if changes were made. The images or other third party material in this article are included in the article's Creative Commons licence, unless indicated otherwise in a credit line to the material. If material is not included in the article's Creative Commons licence and your intended use is not permitted by statutory regulation or exceeds the permitted use, you will need to obtain permission directly from the copyright holder. To view a copy of this licence, visit http://creativecommons.org/licenses/by/4.0/.

(C) The Author(s) 2021 